\title{
WEAKLY CHAIN SEPARATED SETS IN A TOPOLOGICAL SPACE
}

\author{
N. SHEKUTKOVSKI ${ }^{1}$, Z. MISAJLESKI $^{2 *}$, A. VELKOSKA $^{3}$ AND E. DURMISHI $^{4}$ \\ ${ }^{1,2}$ Ss. Cyril and Methodius University. Skopje, North Macedonia \\ ${ }^{3}$ University of Information Science and Technology. Ohrid, North Macedonia \\ ${ }^{4}$ University of Tetovo. Tetovo, North Macedonia \\ *Corresponding author. E-mail: misajleski@gf.ukim.edu.mk
}

\section{DOI: 10.20948/mathmontis-2021-52-1}

Summary. In this paper we introduce the notion of pair of weakly chain separated sets in a topological space. If two sets are chain separated in the topological space, then they are weakly chain separated in the same space. We give an example of weakly chain separated sets in a topological space that are not chain separated in the space. Then we study the properties of these sets. Also we mention the criteria for two kind of topological spaces by using the notion of chain. The topological space is totally separated if and only if any two different singletons (unit subsets) are weakly chain separated in the space, and it is the discrete if and only if any pair of different nonempty subsets are chain separated. Moreover we give a criterion for chain connected set in a topological space by using the notion of weakly chain separateness. This criterion seems to be better than the criterion of chain connectedness by using the notion of pair of chain separated sets. Then we prove the properties of chain connected, and as a consequence of connected sets in a topological space by using the notion of weakly chain separateness.

\section{INTRODUCTION}

The definition of connectedness, that is considered as standard, was first given in the beginning of 20th century by Riesz and Hausdorff.

In 1883 , Cantor gave the definition of connectedness in $\mathbb{R}^{n}$ by using the notion of chain. Later the definition is generalized to all topological spaces: The topological space $X$ is connected if for every $x, y \in X$ and every open covering $\mathcal{U}$ of $X$ there exists a chain in $\mathcal{U}$ that connects $x$ and $y([3,4])$. For more details about connectedness see [5-8].

In papers [1] and [2], rather than as a space, is generalized the notion of connectedness as a set in a topological space that is called a chain connected set.

We will write some definitions and statements from article [1].

By a covering we understand a covering consisting of open sets.

Suppose $X$ is a set, $\mathcal{U}$ is a family of subsets of $X$, and $x, y \in X$. A chain in $\mathcal{U}$ that connects $x$ and $y$ (from $x$ to $y$, from $y$ to $x$ ) is a finite sequence $U_{1}, U_{2}, \ldots, U_{n}$ in $\mathcal{U}$, such that $U_{i} \cap U_{i+1} \neq \varnothing, \quad i=1,2, \ldots, n-1$, and $x \in U_{1}, y \in U_{n}$. In this paper a set is a topological subspace, and a chain is a chain that consists of open sets.

Let $X$ be a topological space and $C \subseteq X$.

Definition 1.1. The set $C$ is chain connected in $X$, if for every covering $\mathcal{U}$ of $X$ in $X$ and every $x, y \in C$, there exists a chain in $\mathcal{U}$ that connects $x$ and $y$.

2010 Mathematics Subject Classification: Primary: 54D05.

Key words and Phrases: General topology, chain, covering, connectedness, chain connectedness, weakly chain separateness. 
So, the space $X$ is connected if and only if $X$ is chain connected in $X$.

Let $X$ be a topological space, and let $A$ and $B$ be nonempty subsets of $X$.

Definition 1.2. The sets $A$ and $B$ are chain separated in $X$, if there exists a covering $\mathcal{U}$ of $X$ in $X$ such that for every point $x \in A$ and every $y \in B$, there is no chain in $\mathcal{U}$ that connects $x$ and $y$.

Theorem 1.1. The sets $A$ and $B$ are separated if and only if $A$ and $B$ are chain separated in $A \cup B$.

Theorem 1.2. The set $C$ is chain connected in $X$, if and only if $C$ cannot be represented as a union of two chain separated sets $A$ and $B$ in $X$

Let $X$ be a topological space and $x, y \in X$.

Definition 1.3. The element $x$ is chain related to $y$ in $X$, and we denote it by $\underset{x}{x} y$, if for every covering $\mathcal{U}$ of $X$ in $X$ there exists a chain in $\mathcal{U}$ that connects $x$ and $y$.

The chain relation in a topological space $X$ is an equivalence relation, and it depends on the set $X$ and the topology $\tau$ of $X$. The chain relation splits the space into classes.

We denote $x_{\mathcal{U}, X}^{\sim} y$, if for the covering $\mathcal{U}$ of $X$ in $X$ there exists a chain in $\mathcal{U}$ that connects $x$ and $y$.

We denote by $A_{X}(x, \mathcal{U})$ the set that consists of all elements $y \in X$ such that $x_{\tilde{\mathcal{U}, X}} y$. The set $A_{X}(x, \mathcal{U})$ is nonempty, open, and closed.

Let $X$ be a topological space, $x \in X$ and $\mathcal{U}$ be a covering of $X$. The infinite star of $x \in X$ and $\mathcal{U}$ in $X$ is denoted by $s t^{\infty}(x, \mathcal{U})[1,9]$.

\section{PAIR OF WEAKLY CHAIN SEPARATED SETS}

Let $X$ be a topological space, and let $A, B \subseteq X$.

Definition 2.1. The nonempty sets $A$ and $B$ are weakly chain separated in $X$, if for every point $x \in A$ and every $y \in B$, there exists a covering $\mathcal{U}=\mathcal{U}(x, y)$ of $X$ in $X$ such that there is no chain in $\mathcal{U}$ that connects $x$ and $y$.

The notion is similar to the notion of pair of chain separated sets in a topological space. Therefore, the analogue theorems to chain separated sets, presented in [1], are valid for weakly chain separated sets. 
From the definition, it follows that:

Proposition 2.1. If $A$ and $B$ are weakly chain separated in $X$, then any pair of nonempty sets $C$ and $D$, where $C \subseteq A$ and $D \subseteq B$, are weakly chain separated in $X$.

The following theorem will show us that two sets, which are weakly chain separated in a space, are also weakly chain separated in every subspace.

Let $X$ be a topological space, let $Y \subseteq X$, and let $A$ and $B$ be nonempty subsets of $Y$.

Theorem 2.1. If $A$ and $B$ are weakly chain separated in $X$, then $A$ and $B$ are weakly chain separated in $Y$.

Proof. Let the sets $A$ and $B$ be weakly chain separated in $X$ and let $x \in A$ and $y \in B$. It follows that there exists a covering $\mathcal{U}$ of $X$ in $X$ such that there is no chain in $\mathcal{U}$ that connects $x$ and $y$. Then

$$
\mathcal{U}_{y}=\mathcal{U} \cap Y=\{U \cap Y \mid U \in \mathcal{U}\}
$$

is covering of $Y$ in $Y$ such that there is no chain in $\mathcal{U}_{Y}$ that connects $x$ and $y$

Remark 2.1. The most important case of the previous theorem is when $Y=A \cup B$.

The definition of pair of chain separated sets, the definition of pair weakly chain separated sets, and the properties of quantifiers, leads to the following statement.

Theorem 2.2. If the sets $A$ and $B$ are chain separated in $X$, then $A$ and $B$ are weakly chain separated in $X$.

The next example shows that the converse statement does not hold in general.

Example 2.1. Let $A=\{0\}, B=\left\{\frac{1}{n} \mid n \in \mathbb{N}\right\}$, and $X=A \cup B$. The sets $A$ and $B$ are weakly chain separated in $X$, but $A$ and $B$ are not chain separated in $X$ i.e. $A$ and $B$ are not separated.

Proof. Let $b \in B$. Then $b=\frac{1}{n_{0}}$ for some $n_{0} \in \mathbb{N}$, and for the covering:

$$
\mathcal{U}=\left\{\left[0, \frac{1}{n_{0}}\right) \cap X,\left\{\frac{1}{n_{0}}\right\},\left\{\frac{1}{n_{0}+1}\right\}, \ldots\right\},
$$

there is no chain in $\mathcal{U}$ that connects 0 and $b$. It follows that $A$ and $B$ are weakly chain separated in $X$.

On the other hand, since the topology on $X$ is relative to $\mathbb{R}$, every element of arbitrary covering of $X$ that contains the point 0 , also contains a point from the set $B$. It follows that $A$ and $B$ are not chain separated in $X$. 
The definition of pair of chain separated sets, the definition of pair weakly chain separated sets, and the criterion of chain separated sets in their union by using the notion of separated sets (see introduction), leads to the following two statements.

Corollary 2.1. If $A$ and $B$ are separated then $A$ and $B$ are weakly chain separated in $A \cup B$.

Theorem 2.3. Singletons $A$ and $B$ are weakly chain separated in $X$ if and only if they are chain separated in $X$

The definition of pair of weakly chain separated sets and the definition of chain relation, lead to the following statement which is a criterion for weakly chain separated sets by using the chain relation. The chain relation has a short notation and therefore we will use this criterion in the proofs.

Proposition 2.2. Two sets $A$ and $B$ are weakly chain separated in $X$ if and only if for every $x \in A$ and $y \in B, \underset{x}{x}+y$

The last proposition in case of chain separateness (remark 4.4 in [1]) is valid in one direction. From the last proposition it follows the next statement:

Corollary 2.2. Let $x, y \in X$. Then $\underset{x}{x} y$, if and only if $X$ cannot be represented as a union of two weakly chain separated sets $A$ and $B$ that contain $x$ and $y$, respectively.

It is easily seen that the next statement is valid:

Theorem 2.4. If the function $f: X \rightarrow\{0,1\}$, such that $f(A)=\{0\}$ and $f(B)=\{1\}$, is continuous then the sets $A$ and $B$ are chain separated (weakly chain separated) in $X$.

According to [1], two nonempty sets $A$ and $B$ are functionally separated in $X$ if there exists a continuous function $f: X \rightarrow\{0,1\}$ such that $f(A)=\{0\}$ and $f(B)=\{1\}$. So, the next statement is valid.

Corollary 2.3. If $A$ and $B$ are functionally separated in $X$ then $A$ and $B$ are chain separated (weakly chain separated) in $X$.

From the last corollary it follows that if $A$ and $B$ are functionally separated in $A \cup B$ then $A$ and $B$ are weakly chain separated in $A \cup B$. This statement in the case of chain separateness (corollary 4.3 in [1]) is valid in both direction.

Corollary 2.4. If $f: X \rightarrow[0,1]$ is a continuous function such that $f(A)=\{0\}$ and $f(B)=\{1\}$, then the sets $A$ and $B$ are chain separated (weakly chain separated) in $f^{-1}(0) \cup f^{-1}(1)$ 
From the last corollary it follows that if $f: X \rightarrow[0,1]$ is a continuous function such that $f(A)=\{0\}$ and $f(B)=\{1\}$, then the sets $A$ and $B$ are chain separated (weakly chain separated) in $A \cup B$. The reverse claim does not have to be valid.

Example 2.2. Let $X=[-1,1], A=[-1,0)$ and $B=(0,1]$. Then sets $A$ and $B$ are weakly chain separated in $A \cup B$, but there is no continuous function $f: X \rightarrow[0,1]$ such that $f(A)=\{0\}$ and $f(B)=\{1\}$.

At the end we mention the criteria for two kind of topological spaces by using the notion of chain.

Let $X$ be a topological space.

Theorem 2.5. The space $X$ is the discrete if and only if any two disjoint nonempty subsets of $X$ are chain separated in $X$.

Proof. Let the space $X$ be the discrete space and let $x, y \in X, x \neq y$. Then for the covering $\mathcal{U}=\{\{x\}, X \backslash\{x\}\}$ it follows that $\underset{\mathcal{U}, X}{x} y$ i.e. $\{x\}$ and $\{y\}$ are chain separated in $X$.

Let any two disjoint subsets of $X$ be chain separated in $X$, and let $x \in X$. Then $\{x\}$ is open set, since otherwise $\{x\}$ and $X \backslash\{x\}$ will not be chain separated.

So, $X$ is the discrete if and only if for every $A, B \subseteq X$ such that $A, B \neq \varnothing$ and $A \cap B=\varnothing$, there exist a covering $\mathcal{U}$ of $X$, such that for every $x \in A$ and every $y \in B$ there is no chain in $\mathcal{U}$ that connects $x$ and $y$. From the last theorem it follows that we can prove the properties of the discrete space by using the notion of chain.

Theorem 2.6. The space $X$ is totally separated if and only if any two disjoint singletons subsets are weakly chain separated in $X$.

Proof. $(\Rightarrow)$ Let $X$ be totally separated and let $x, y \in X, x \neq y$. Since $X$ is totally separated, then there is an open and closed subset $U \subseteq X$ such that $x \in U$ and $y \in X \backslash U$. Then for the covering $\mathcal{U}=\{U, X \backslash U\}$ it follows that $\underset{\mathcal{U}, X}{x} y$, hence $\underset{X}{x} y$.

$(\Leftarrow)$ Let any two different singletons be weakly chain separated and let $x, y \in X, x \neq y$. Since $x \underset{X}{x} y$, then there exists a covering $\mathcal{U}$ such that there is no chain in $\mathcal{U}$ that connects $x$ and $y$. Then the sets $A=A_{X}(x, \mathcal{U})$ and $X \backslash A$ are open and closed in $X$ such that $x \in A$ and $y \in X \backslash A$. It follows that $X$ is totally separated.

At the end of the section we generalise the notion of totally separated space by using the notion of chain, to a set in a topological space.

Let $X$ be a topolgical space and let $A$ be a subspace of $X$. 
Definition 2.2. The set $A$ is totally weakly chain separated in $X$ if any two disjoint singletons subsets of $A$ are weakly chain separated in $X$.

So, the set $A$ is totally weakly chain separated in $X$ if for every $x, y \in A$ there exists a covering $\mathcal{U}$ of $X$ such that there is no chain in $\mathcal{U}$ that connects $x$ and $y$.

\section{STRONGLY CHAIN CONNECTED SETS}

Let $X$ be a topological space, and $C \subseteq X$.

Definition 3.1. A set $C$ is strongly chain connected in $X$ if $C$ cannot be represented as a union of two weakly chain separated sets $A$ and $B$ in $X$.

From the definition it follows that a space $X$ is strongly chain connected in $X$ if it cannot be represented as a union of two weakly chain separated sets $A$ and $B$ in $X$.

Since the property of weak chain connectedness is weaker then the property of chain connectedness, we expect the property of strong chain connectedness to be stronger then the property of chain connectedness. Actually, the next theorem shows that they are equivalent.

Theorem 3.1. The set $C$ is strongly chain connected in $X$ if and only if $C$ is chain connected in $X$.

Proof. $(\Rightarrow)$ If the set $C$ is not chain connected in $X$, then there exists a pair of chain separated sets $A$ and $B$ in $X$, such that $C=A \cup B$. But then $A$ and $B$ are weakly chain separated in $X$ i.e. $C$ is not strongly chain connected in $X$.

$(\Leftarrow)$ If $C$ is not strongly chain connected in $X$ i.e. $C$ can be represented as a union of two weakly chain separated sets $A$ and $B$ in $X$ it follows that there exist $x, y \in C$ and a covering $\mathcal{U}$ of $X$ in $X$ such that there is no chain in $\mathcal{U}$ that connects $x$ and $y$. Therefore $C$ is not chain connected in $X$.

Remark 3.1. The most important case of the theorem is when $C=X$.

From the last definition it follows that if the set $C$ is strongly chain connected in $X$ then for every covering $\mathcal{U}$ of $X$ in $X$ and every $x, y \in C$, there is no chain in $\mathcal{U}$ that connects $x$ and $y$.

\section{PROPERTIES OF STRONGLY CHAIN CONNECTED SET}

In this section we reformulate the statements from [1], by changing the notion of chain connected set with strongly chain connected set and we rewrite some of the proofs to be more readable. In the proofs we use the new chain sonnected criterion by using weakly chain separated sets or we use the short notation of chain connected relation. We also formulate and prove some analogoues statements by changing the notion of chain separated (case of chain separateness) with weakly chain separated sets (case of weakly chain separateness).

The next proposition follows from the definition of strongly chain connected set in a topological space. 
Proposition 4.1. If the set $C$ is strongly chain connected in $X$, then each subset of $C$ is strongly chain connected in $X$

Let $X$ be a topological space and $C \subseteq Y \subseteq X$.

Theorem 4.1. If the set $C$ is strongly chain connected in $Y$, then $C$ is strongly chain connected in $X$.

Proof. If the set $C$ is not strongly chain connected in $X$ then $C$ can be represented as a union of two weakly chain separated sets $A$ and $B$ in $X$. From theorem 2.3. it follows that $A$ and $B$ are weakly chain separated sets in $Y$ i.e. $C$ is not strongly chain connected in $Y$

Remark 4.1. The most important case of the previous theorem is when $C=Y$.

Example 4.1. Let $X=[-1,1]$ and $Y=[-1,0) \cup(0,1]$. Then $Y$ is strongly chain connected in $X$, but it is not strongly chain connected in $Y$. Moreover $Y$ is not connected.

From the theorem 3.1 it follows that:

Corollary 4.1. The set $C$ is strongly chain connected in $X$ if and only if for every $x, y \in C, \underset{x}{x} y$

Remark 4.2. The most important case of the previous corollary is when $C=X$

Therefore, $C$ is not strongly chain connected in $X$ if and only if there exist $x, y \in C$ such that $x+\underset{x}{x} y$.

Theorem 4.2. A space $X$ is connected if and only if $X$ is strongly chain connected in $X$.

Proof. If $X$ is empty or a singleton, then $X$ is connected and strongly chain connected in $X$. Let $X$ be composed of at least two elements.

$(\Rightarrow)$ If $X$ is not strongly chain connected in $X$ i.e., from theorem 3.1, it is not chain connected in $X$ then it follows that there exists a covering $\mathcal{U}$ of $X$ and there exist elements $x, y \in X$, such that $\underset{\mathcal{U}, X}{x} y$. Hence $A$ and $X \backslash A$ where $A=A_{X}(x, \mathcal{U})$ are nonempty, open and closed sets whose union is $X$. It follows that $X$ is not connected.

$(\Leftarrow)$ If $X$ is not connected then $X$ can be represented as a union of two open and closed sets $A$ and $B$. Hence $\{A, B\}$ is covering of $X$ such that for every $x \in A$ and $y \in B, x+y$, i.e. $A$ and $B$ are chain separated sets in $X$. Therefore $A$ and $B$ are weakly chain separated sets in $X$ i.e. $X$ is not strongly chain connected in $X$

The last theorem allows us to prove the properties of connected spaces by using the notion of weakly chain connectedness.

Notice that, as a consequence, it follows that if $C$ is connected, then $C$ is strongly chain connected in every super space $X$. In addition, a topological space $C$ is strongly chain connected in $C$, if and only if it cannot be represented as a union of two separated sets. 
Let $X$ be a topological space and $C \subseteq Y \subseteq X$.

Theorem 4.3. Let $X=A \cup B$, where $A$ and $B$ are weakly chain separated sets in $X$, and $C$ is a strongly chain connected set in $X$. Then $C \subseteq A$ or $C \subseteq B$.

Proof. Let $C$ be a strongly chain connected set in $X$, i.e. for every $x, y \in C, \underset{X}{x} y$.

If there exist $x \in A \cap C$ and $y \in B \cap C$, since $A$ and $B$ are weakly chain separated sets in $X$, it follows that $x+y$, which contradicts the assumption. So, $C \subseteq A$ or $C \subseteq B$.

A direct consequence of the last theorem are the next two corollaries:

Corollary 4.2. Let $X=A \cup B$, where $A$ and $B$ are chain separated sets in $X$, and $C$ is a strongly chain connected set in $X$. Then $C \subseteq A$ or $C \subseteq B$.

Corollary 4.3. Let $X=A \cup B$, where $A$ and $B$ are separated. If $C$ is a connected set, then $C \subseteq A$ or $C \subseteq B$

The next theorem and its remark show that chain relation and strongly chain connected set is invariant with respect to continuous function.

Theorem 4.4. Let $x, y \in X$. If $\underset{X}{\underset{\sim}{\sim} y}$ and $f: X \rightarrow Y$ is a continuous function, then $f(x) \underset{f(X)}{\sim} f(y)$.

Proof. The function $f: X \rightarrow Y$ is continuous if and only if $f: X \rightarrow f(X)$ is continuous.

Let $f(x), f(y) \in f(X)$ and $\mathcal{V}$ be a covering of $f(X)$.

Then $\mathcal{U}=f^{-1}(\mathcal{V})$ is a covering of $X$ and since $x_{X}^{\sim} y$, there exists a chain

$$
f^{-1}\left(V_{1}\right), f^{-1}\left(V_{2}\right), \ldots, f^{-1}\left(V_{n}\right)
$$

in $\mathcal{U}$ that connects $x$ and $y$. Since $f^{-1}\left(V_{i}\right) \cap f^{-1}\left(V_{i+1}\right) \neq \varnothing, i=1,2, \ldots, n-1$, it follows that

$$
\varnothing \neq f\left(f^{-1}\left(V_{i}\right)\right) \cap f\left(f^{-1}\left(V_{i+1}\right)\right)=V_{i} \cap V_{i+1}, i=1,2, \ldots, n-1,
$$

i.e. $V_{1}, V_{2}, \ldots, V_{n}$ is a chain in $\mathcal{V}$ that connects $f(x)$ and $f(y)$. Therefore $f(x)_{f(X)} f(y)$.

Corollary 4.4. If $C$ is strongly chain connected in $X$ and $f: X \rightarrow Y$ is a continuous function, then $f(C)$ is strongly chain connected in $f(X)$

The next well known result is a consequence of the last corollary:

Remark 4.3. Let $C \subseteq X$. If $C$ is a connected set and $f: X \rightarrow Y$ is a continuous function, then $f(C)$ is connected. 
Theorem 4.5. If $C \subseteq D \subseteq \bar{C} \subseteq X$. The set $C$ is strongly chain connected in $X$, if and only if $D$ is strongly chain connected in $X$.

Proof. Clearly, if $D$ is strongly chain connected in $X$ then proposition 4.1 implies that $C$ is strongly chain connected in $X$.

Let $C$ be strongly chain connected in $X$, let $x, y \in D$, and let $\mathcal{U}$ be a covering of $X$. Since $D \subseteq \bar{C}$, members $U$ and $V$ of $\mathcal{U}$ that contain $x$ and $y$, also contain some elements $x_{1} \in C$ and $y_{1} \in C$, respectively. Then $\underset{x}{\sim} \underset{x}{x_{1}}, x_{1} \underset{X}{\sim} y_{1}$, and $y_{1} \underset{X}{\sim} y$, i.e. $\underset{x}{\sim} y$. It follows that $D$ is strongly chain connected in $X$

Remark 4.4. The most important case of the theorem is when $D=\bar{C}$.

As a consequence, an analogous statement for connected sets holds, but only in one direction.

Corollary 4.5. Let $X$ be a topological space and $C \subseteq X$. If $C$ is a connected set and $C \subseteq D \subseteq \bar{C}$, then $D$ is connected.

Proof. If $C$ is a connected set i.e. $C$ is strongly chain connected in $C$ it follows that $C$ is strongly chain connected in $D$ and, by the last theorem, $D$ is strongly chain connected in $D$ i.e. $D$ is connected.

Lemma 4.1. Let $C, D \subseteq X$. If $C$ and $D$ are strongly chain connected in $X$ and $\bar{C} \cap \bar{D} \neq \varnothing$, then the union $\bar{C} \cup \bar{D}$ is strongly chain connected in $X$.

Proof. Let $x, y \in \bar{C} \cup \bar{D}$ and let $z \in \bar{C} \cap \bar{D}$. Since $C$ and $D$ are strongly chain connected in $X$, by using the previous theorem it follows that $\underset{X}{\sim} \underset{X}{z}$ and $\underset{X}{\sim} y$, hence $x_{X} \underset{\sim}{\sim} y$

Theorem 4.6. Let $C_{i}, i \in I$ be a family of strongly chain connected subsets of $X$. If there exists $i_{0} \in I$ such that for every $i \in I, \bar{C}_{i_{0}} \cap \bar{C}_{i} \neq \varnothing$, then the union $\bigcup_{i \in I} \bar{C}_{i}$ is chain connected in $X$.

Proof. Let $x, y \in \bigcup_{i \in I} \bar{C}_{i}$, i.e. $x \in \bar{C}_{x}$ and $y \in \bar{C}_{y}$ for some $x, y \in I$. Let $x_{1} \in \bar{C}_{i_{0}} \cap \bar{C}_{x}$ and $y_{1} \in \bar{C}_{i_{0}} \cap \bar{C}_{y}$. Then $\underset{X}{\sim} x_{1}, x_{1} \underset{X}{\sim} y_{1}$, and $\underset{y_{1}}{\underset{X}{\sim}} y$. It follows that $\underset{X}{x} y$

From the theorem it follows that if $C_{i}, i \in I$ is a family of strongly chain connected subsets of $\bigcup_{i \in I} C_{i}$, then the union $\bigcup_{i \in I} C_{i}$ is chain connected in $\bigcup_{i \in I} C_{i}$. A direct consequence of the last statement is:

Corollary 4.6. Let $C_{i} \subseteq X, i \in I$ be a family of connected sets. If there exists $i_{0} \in I$ such that for every $i \in I, C_{i_{0}} \cap C_{i} \neq \varnothing$, then the union $\bigcup_{i \in I} C_{i}$ is connected. 
Note that we can not use the assumption $\bar{C}_{i_{0}} \cap \bar{C}_{i} \neq \varnothing$ in the last corollary since from $\bar{C}_{i_{0}} \cap \bar{C}_{i} \neq \varnothing$ in $X$ we cannot conclude that $\bar{C}_{i_{0}} \cap \bar{C}_{i} \neq \varnothing$ in $\bigcup_{i \in I} C_{i}$.

It is clear that if every two points $x$ and $y$ of $X$ are in a strongly chain connected set $C_{x y}$ in $X$, then $X$ is strongly chain connected. The next corollary follows.

Corollary 4.7. If for every two points $x$ and $y$ of $X$ there exists a connected set $C_{x y}$ in $X$ containing them, then $X$ is connected.

Theorem 3.7 from [1] is not walid in the case of weakly chain separateness.

Theorem 4.7. Set $C$ is strongly chain connected in $X$, if and only if for every $x \in C$ and every covering $\mathcal{U}$ of $X, C \subseteq s t^{\infty}(x, \mathcal{U})$

Corollary 4.8. Space $X$ is connected, if and only if for every $x \in X$ and every covering $\mathcal{U}$ of $X, X=s t^{\infty}(x, \mathcal{U})$.

In the next theorem we will give a strong chain connectedness criterion using continuous function.

Theorem 4.8. A space $X$ is strongly chain connected, if and only if every continuous function $f: X \rightarrow\{0,1\}$ is constant.

As a consequence, it follows that $X$ is strongly chain connected, if and only if there is no continuous function $f: X \rightarrow[0,1]$, such that $f(A)=\{0\}$ and $f(B)=\{1\}$ for every nonempty pair of sets $A$ and $B$ such that $A \cup B=X$.

Let $X$ be a topological space and $x \in C \subseteq X$.

Definition 4.1. The strongly chain connected component of the point $x$ of $C$ in $X$, denoted by $V_{C X}(x)$, is the biggest chain connected subset of $C$ in $X$ that contains $x$.

From the last definition and the definition of chain relation follow the next three statements:

Proposition 4.2. The strongly chain connected component $V_{C X}(x)$ of the point $x$ of $C$ in $X$ is the set of all points $y \in C$ such that $\underset{X}{x} y$

Proposition 4.3. The set of all strongly chain connected subsets of $C$ in $X$ consist of all strongly chain connected components of $C$ in $X$ and their subsets. 
Proposition 4.4. For every $x \in C, V_{C X}(x)=C \cap V_{X X}(x)$. Each strongly chain connected component of $X$ in $X$ contains at most one strongly chain connected component of $C$ in $X$.

Since the chain relation is an equivalence relation, from the last proposition it follow the next two statements.

Proposition 4.5. Let $x, y \in C$. If $y \in V_{C X}(x)$, then $V_{C X}(x)=V_{C X}(y)$.

Proposition 4.6. Let $x, y \in C$. If $V_{C X}(x) \neq V_{C X}(y)$, then $V_{C X}(x) \cap V_{C X}(y)=\varnothing$.

As a consequence of the definition of strongly chain connected component and the last two statemenents, the next proposition is valid.

Proposition 4.7. For every $x \in C$,

$$
V_{C C}(x) \subseteq V_{C X}(x)=\bigcup_{y \in V_{C X}(x)} V_{C C}(y)
$$

The proposition shows that every strongly chain connected component of $C$ in $X$ is a union of strongly chain connected components of $C$ in $C$.

Proposition 4.8. The strongly chain connected components of $X$ in $X$ are closed sets, i.e. for every $x \in X, V_{X X}(x)=\overline{V_{X X}(x)}$.

Proof. Let $y \in \overline{V_{X X}(x)}$ and $\mathcal{U}$ be a covering of $X$. Then there exists a neighbourhood

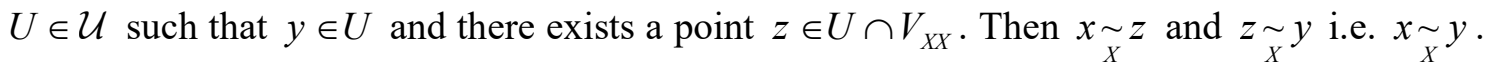
So, $y \in V_{X X}(x)$ i.e. $V_{X X}(x)$ is closed set.

Proposition 4.9. Let $x \in X$ and $C(x)$ be a connected component of $X$. Then $C(x) \subseteq V_{X X}(x)$

Quasicomponent of the element $x$ in a topological space $X$, denoted with $Q_{X}(x)$, is the intersection of all clopen (closed and open) sets in $X$ that contain $x$.

Theorem 4.9. Quasicomponents and strongly chain connected components in a topological space $X$ coincide, i.e., for every $x \in X, Q_{X}(x)=V_{X X}(x)$.

Proof. $(\Leftarrow)$ If $y \notin Q_{X}(x)$, since $Q_{X}(x)$ is the quasicomponent of $x$, it follows that $y \notin A$ for some open and closed set $A$ such that $Q_{X}(x) \subseteq A$. But then for the covering $\mathcal{U}=\{A, X / A\}$, it follows that $\underset{\mathcal{U}, X}{x} \underset{\text {, }}{x}$, and hence $x+\underset{X}{+} y$ i.e. $y \notin V_{X X}(x)$.

$(\Rightarrow)$ If $y \notin V_{X X}(x)=\bigcap_{\mathcal{U} \in \operatorname{Cov}(X)} A_{X}(x, \mathcal{U})$ where $\operatorname{Cov}(x)$ consists of all coverings of $X$ it follows that $y \notin A=A_{X}(x, \mathcal{U})$ where $\mathcal{U}$ is some covering of $X$. Since $A$ is an open and closed set that contain $x$, it follows that $Q_{X}(x) \subseteq A$. Then $y \notin Q_{X}(x)$. 
From the last theorem it follows that quasicomponent of the point $x$ is the biggest strongly chain connected set in $X$ that contains $x$.

There exists a criterion for quasicomponenet by using the notion of chain: The quasicomponent of the point $x$ in $X$ consits of all $y \in X$ such that for every covering $\mathcal{U}$ of $X$ there exists a chain in $\mathcal{U}$ that connects $x$ and $y$ [1]. Hence the quasicomponent of $x$ in $X$ consists of all $y \in X$ such that $x \sim y$. Therefore the propositions 4.8 and 4.9 and the theorem 4.9 are reformulations of two properties and a criterion of the quasicomponents.

The next proposition is the summary of the propositions 4.8, 4.9, and the theorem 4.9.

Proposition 4.10. For every $x \in C$,

$$
Q_{C}(x)=V_{C C}(x) \subseteq \bigcup_{y \in V_{C X}(x)} Q_{C}(y)=V_{C X}(x) \subseteq V_{X X}(x)=Q_{X}(x)
$$

So strongly chain connected components of a set in a topological space are a union of quasicomponents of the set i.e. for every $x \in C$,

$$
V_{C X}(x)=\bigcup_{y \in V_{C X}(x)} Q_{C}(y)
$$

and if the set agrees with the space, the strongly chain connected components match with the quasicomponents.

\section{CONCLUSIONS}

In this work we defined a pair of weakly chain separated sets, and strongly chain connected set in a topological space, and studied the properties of these sets. As a consequence, by using the notion of weakly chain separateness i.e. a chain, the properties of connected sets are proven.

\section{REFERENCES}

[1] Z. Misajleski, N. Shekutkovski, and A. Velkoska, "Chain Connected Sets In A Topological Space", Kragujevac Journal of Mathematics, 43 (4), 575-586 (2019).

[2] N. Shekutkovski, Z. Misajleski, and E. Durmishi, "Chain Connectedness", AIP Conference Proceedings, 2183, 030015-1-030015-4 (2019).

[3] N. Shekutkovski, "On the concept of connectedness", Matematichki bilten, 40 (1), 5-14 (2016).

[4] Alessandro Berarducci, Dikran Dikranjan, and Jan Pelant, "Uniform quasicomponents, thin spaces and compact separation", Topology and its applications, 122, 51-64 (2002).

[5] R.Engel'king, Obshchaya topologiya, Mir, Moskva (1986).

[6] J.G. Hocking and G.S. Young, Topology, Addison-Wesley, Reading, Massachusetts (1961).

[7] J. Munkres, Topology, Pearson Education (2014).

[8] K. Kuratovskii, Topologiya, Mir, Moskva, Tom 1, (1966), Tom 2, (1969).

[9] S.A. Naimpally and C.M. Pareek, "Of the compactness of connected sets", Rev. Un. Mat. Argentina, 39, 45-52 (1994).

Received September 24, 2021 